\title{
The effect of different psychophysical methods on visual orientation during tilt
}

\author{
N. J. WADE, ${ }^{1}$ Monash University, Clayton, \\ Victoria, Australia
}

The visual vertical with 30 deg right head tilt was determined by three psychophysical procedures: the methods of adjustment with and without bracketing and a modified up-and-down (staircase) method. The visual vertical was on the opposite side of the gravitational vertical to tilt (E-effect) under all conditions. However, the E-effect measured by the up-and-down method was significantly larger than the combined average of the two methods of adjustment. The methods of adjustment with and without bracketing yielded approximately the same means for the visual vertical when averaged over starting positions, but differed significantly in the linear trends taken over starting positions. $A$ greater error of anticipation resulted when bracketing was not allowed.

Experiments concerned with visual orientation during tilt have employed widely differing methodologies. They have, nonetheless, yielded results that are in general agreement. In the absence of any visual surround cues and with the head upright, the visual vertical is in close correspondence to the gravitational vertical. However, during lateral tilt, the visual vertical is systematically displaced depending upon the degree of tilt. For body tilts less than about $60 \mathrm{deg}$, the visual vertical is on the opposite side of the gravitational vertical to tilt, whereas the reverse occurs for greater degrees of tilt (Bauermeister, 1964; Miller, Fregly, \& Graybiel, 1968; Schöne, 1964). These are referred to as the E- and A-effects, respectively. Head tilt relative to the upright trunk produces only the E-effect, since its limit is about $40 \mathrm{deg}$ (Wade, 1969b).

The two psychophysical procedures most commonly used to determine the visual vertical have been adjustment by a modified method of limits and the method with $S$ controlling the line orientation and making bracketing adjustments around the visual vertical. The modified method of limits is essentially similar to the method of adjustment controlled by $S$ but without bracketing, because neither allows fine adjustment around the vertical. The method of constant stimulus presentation or any of its variants have rarely been employed. Egocentric visual orientation during tilt has been compared with the modified method of limits and the method of constant stimuli (Bauermeister, Wapner, \& Werner, 1967). Different values for the visual alignment to the body median plane were obtained such that it was closer to the objective median plane with the constants procedure.

The present experiment was designed to compare the visual vertical derived from the methods of adjustment with and without bracketing and, in turn, to compare these with that measured by an up-and-down (staircase) method at one head tilt only. For the up-and-down method, a two-category forced-choice procedure was used, together with a double staircase (Cornsweet, 1962). As a further modification, two step sizes were employed in the staircase procedure. The first, coarser one started from the gravitational vertical, and the second, finer step size commenced at the mean derived from the first one (Wetherill, 1963).

$$
\text { APPARATUS }
$$

The head position was controlled by means of side head clamps and an individual dental composition bite board. These could be rotated about a horizontal axis to achieve lateral head tilt. The stimulus line $(0.30 \mathrm{fL})$ corresponded to a 154-mm-long and 0.6-mm-wide aperture in an otherwise light-tight lamp housing, $180 \mathrm{~cm}$ away from S's eyes, with its center at eye level and in the median plane. A switching device was attached to the light arrangement so that the line could be presented briefly, by means of a pulse from a decade timer, or continuously. The line could be rotated by $S$, using a switch by his right hand, which activated a 1-rpm reversible motor. For the up-and-down method, a response switch was located in S's body midline and could be pressed to the right or left, so lighting one of two globes visible only to $\mathrm{E}$.

$$
\text { SUBJECTS }
$$

Twenty-four Ss from an introductory course in psychology participated.

$$
\text { PROCEDURE }
$$

Only 30-deg right head tilt was investigated. Each $\mathrm{S}$ made visual vertical judgments using a method of adjustment with bracketing, a method of adjustment with no bracketing, and an up-and-down method. The six possible orders of the three methods were counterbalanced over the $24 \mathrm{Ss}$, and each $\mathrm{S}$ was tested under the three conditions in a single session.

For the bracketing method of adjustment, $\mathbf{S}$ was instructed to move the line of light by means of the switch so that it was rotated initially beyond the vertical position, reversed in direction to beyond the vertical again, and then adjusted finely until the visual vertical was located. Two practice trials were given with the head upright to ensure that the instructions had been understood. Six adjustments with right head tilt were made to the visual vertical from starting positions of 10,20 , and $30 \mathrm{deg} \mathrm{CW}$ and $\mathrm{CCW}$, presented in random order. The head was returned to the upright position after completion of each adjustment and remained there for $45 \mathrm{sec}$ before the next tilt.

For the no-bracketing method of adjustment, $S$ was instructed to move the line directly to the vertical position and to stop it there. No further adjustment of the line was allowed after it had been stopped. The same procedure was then employed as for the bracketing trials.

With the up-and-down method, $S$ was instructed that the line would appear briefly at one position only and that he had to report whether the top of the line was tilted to the left or right by pressing the switch to the left or right, respectively. It was stressed that a choice had to be made after each presentation. The presentation method was demonstrated in a lighted room and two practice trials were given in the darkened room with the head upright. During head tilt, $S$ opened his eyes at an auditory signal, the line was presented for $0.15 \mathrm{sec}, S$ indicated the direction of visual tilt of the line, and then closed his eyes. The head was returned to the upright and remained in that position for $30 \mathrm{sec}$ before the next tilt. This procedure was repeated until the stopping rule for the up-and-down method was satisfied. Two independent, randomly alternating staircases were started from the gravitational vertical and proceeded with step sizes of $4 \mathrm{deg}$ until six reversals had been obtained, i.e., six changes from a left to a right response or vice versa. The mean of the six reversals was computed and two independent staircases were started from the nearest integer degree to this mean and progressed with step sizes of $2 \mathrm{deg}$. The stopping rule was six reversals with the 2-deg step size.

The head was tilted more times with the up-and-down method than with the two adjustment methods, but the time involved in each tilt was shorter because the line was transiently presented.

\section{RESULTS AND DISCUSSION}

The individual mean visual verticals for the two methods of adjustment were computed by averaging over starting positions. The visual vertical for the up-and-down method was the mean of the six reversals on the 2-deg step size only. 
Table 1

Means (Degrees) and Variances for Visual Vertical with 30-Deg Right Head Tilt Derived from the Different Psychophysical Methods

\begin{tabular}{lccc}
\hline & \multicolumn{2}{c}{ Psychophysical } & Method \\
\cline { 2 - 3 } & \multicolumn{2}{c}{ Adjustment } & Up-and- \\
\cline { 3 - 4 } & Bracketing & No Bracketing & Down \\
\hline $\mathrm{M}$ & -4.08 & -4.47 & -8.56 \\
$\mathrm{~s}^{2}$ & 12.84 & 13.02 & 25.03 \\
\hline
\end{tabular}

Note-Indicates a $\mathrm{CCW}$ deviation from the gravitational vertical.

This has been shown to give an accurate estimate of the $50 \%$ point of the psychometric function (Wetherill, 1963). Table 1 gives the means and variances for the visual vertical derived from the different psychophysical methods. The visual vertical using the method of adjustment with bracketing was not significantly different from that without bracketing $(F=0.20, \mathrm{df}=1 / 46, \mathrm{p}>.05)$. On the other hand, the visual vertical measured by the up-and-down method did differ significantly from the average of the two methods of adjustment $(F=31.63$, $\mathrm{df}=1 / 46, \mathrm{p}<.05)$.

The values for the visual vertical with the two methods of adjustment at each starting position are shown graphically in Fig. 1. The methods of adjustment combined had a significant linear trend $(F=68.32$, df $=1 / 23, p<.05)$, but they differed significantly from one another in terms of the linear trend over starting positions $(F=41.94$, df $=1 / 23, p<.05)$.

The E-effect occurred with 30-deg right head tilt for all three psychophysical procedures employed, and this was indexed by the $\mathrm{CCW}$ visual vertical. The E-effect was approximately the same for the two methods of adjustment when averaged over starting positions, although it was slightly greater when bracketing was not allowed. With the up-and-down method, however, the E-effect was significantly larger than that with either of the other two methods, and the variance was also higher. It is considered that three factors may have contributed to this difference. Firstly, proprioceptive adaptation of the neck system during the short periods of head tilt may have differed. Such adaptation has been shown to influence visual orientation following a period of head tilt (Day \& Wade, 1966), and would be similarly expected to reduce the magnitude of the E-effect during tilt. ${ }^{2}$ With the adjustment methods, the head was tilted for longer than with the up-and-down procedure, and so there may have been a greater degree of proprioceptive adaptation with a consequent reduction in the E-effect. However, the difference in head-tilt luration was short in relation to the temporal development of such adaptation (Wade \& Day, 1968) and, therefore, this is unlikely to have been a major factor contributing to the difference between the various procedures. Secondly, the longer viewing times associated with the methods of adjustment may have resulted in more eye movements over the line. The results of previous experiments (Wade, 1969a) suggest that such scanning eye movements may reduce the E-effect, and the difference in the present experiment is consonant with this interpretation. Thirdly, for the adjustment methods, the asymmetry of the line starting positions with respect to the visual vertical may have reduced the E-effect. The mean visual vertical was $\mathrm{CCW}$ and the starting positions were counterbalanced around the gravitational vertical. This may have biased the visual vertical in the $C W$ direction relative to the up-and-down method in which the line starting position was not a factor during a judgment.

The combined methods of adjustment showed a linear trend over starting positions such that the final location of the visual vertical was closer to the initial position of the line than the mean over starting positions, i.e., there was an error of anticipation. The starting position effect was much greater when bracketing was not allowed, and the linear trend components differed significantly. This result is essentially similar to that found for judgments of the visual vertical with head upright using the two adjustment methods (O'Connell, Lathrop, Weintraub, \& McHale, 1967), although the starting position effects were much greater during head tilt. Linear regression to the starting position has also been demonstrated for a visual spatial aftereffect using the method of adjustment without bracketing (Wenderoth, Curthoys, \& Rodger, 1968).

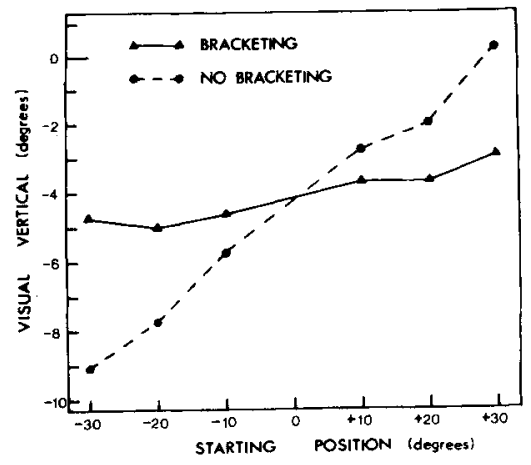

Fig. 1. Visual vertical with 30 -deg right head tilt as a function of line starting position for the methods of adjustment with and without bracketing. (+ denotes a CW deviation from the gravitational vertical; - denotes $\mathrm{CCW}$.)
The influence of the line starting position on visual orientation during tilt has been considered to support sensory-tonic theory by Wapner \& Werner (1957). They state, "We assume that the subject adapts himself to a certain degree to the position in which the stimulus object (rod, etc.) was started at the beginning of the trial [Wapner \& Werner, 1957, p. 11]." This assumption has been rendered untenable for verticality judgments with the head upright (O'Connell et al, 1967; Weintraub, O'Connell, \& McHale, 1964) and requires severe qualification for judgments during tilt. Wapner and Werner employed a modified method of limits which was similar to the method of adjustment without bracketing used in the present experiment. The large errors of anticipation found with this method were greatly reduced when fine adjustment to the visual vertical was permitted. Therefore, it appears that the large effects of the initial line position on the visual vertical found by Wapner and Werner may be attributed to the specific psychophysical method they employed.

It is concluded that the psychophysical procedure used to determine the visual vertical during tilt significantly influences its location. The up-and-down method produced a larger E-effect than the combined average of the two methods of adjustment for 30-deg head tilt. It is considered that differences in eye movements and the asymmetry of the line starting positions associated with the latter methods may have contributed to this difference. The methods of adjustment with and without bracketing yielded approximately the same means for the visual vertical when averaged over starting positions. However, the two methods differed significantly in the linear trend taken over starting positions, such that the visual vertical was closer to the starting position when bracketing was not permitted.

\section{REFERENCES}

BAUERMEISTER, M. Effect of body tilt on apparent verticality, apparent body position, and their relation. Journal of Experimental Psychology, 1964, 67, 142-147.

BAUERMEISTER, M., WAPNER, S., \& WERNER, H. Method of stimulus presentation and apparent body position under lateral body tilt. Perceptual \& Motor Skills, 1967, 24, 43-50.

CORNSWEET, T. N. The staircase method in psychophysics. American Journal of Psychology, 1962, 75, 485-491.

DAY, R. H., \& WADE, N. J. Visual spatial aftereffect from prolonged head-tilt. Science, $1966,154,1201-1202$.

MILLER, E. F., FREGLY, A. R., \& GRAYBIEL, A. Visual horizontal-perception in relation to otolith-function. American Joumal of Psychology, 1968, 81, 488-496.

O'CONNELL, D. C., LATHROP, R. G., 
WEINTRAUB, D. J., \& MchaLE, T. J. Apparent verticality: Psychophysical error versus sensory-tonic theory. Journal of Experimental Psychology, 1967, 73, 347-353.

SCHONE, $H$. On the role of gravity in human spatial orientation. Aerospace Medicine, 1964, 35, 764-772.

WADE, N. J. The effect of stimulus line variations on visual orientation with head upright and tilted. Australian Joumal of Psychology, 1969a, 21, 177-185.

WADE, N. J. Visual orientation as a function of head tilt. Perceptual \& Motor Skills, 1969b, 29, 573-574.

WADE, N. J., \& DAY, R. H. Development and dissipation of a visual spatial aftereffect from prolonged head tilt. Journal of Experimental Psychology, 1968, 76, 439-443.

WAPNER, S., \& WERNER, H. Perceptual development. Worcester, Mass: Clark University Press, 1957.

WEINTRAUB, D. J., O'CONNELL, D. C., \& McHALE, T. J. Apparent verticality: Fundamental variables of sensory-tonic theory reinvestigated. Joumal of Experimental Psychology, 1964, 68, 550-554.

WENDEROTH, P. M., CURTHOYS, I. S., \& RODGER, R. S. Confounding of psychophysical errors and sensory effects in adjustment measures of spatial aftereffects. Perception \& Psychophysics, 1968, 4, 133-138.

WETHERILI, G. B. Sequential estimation of quantal response curves. Joumal of the Royal Statistical Society, 1963, 25, 1-48. NOTES

1. Present address: Max-Planck-Institut : für Verhaltensphysiologie, 8131 Seewiesen, Germany.

2. Unpublished data indicate that changes in visual orientation during prolonged head tilt have similar spatial and temporal characteristics to those following tilt. That is, the E-effect for 30-deg head tilt is reduced by about 2 deg with prolonged tilt, and this adaptation develops within the first few minutes of tilt. Furthermore, the adaptation effect measured during tilt was similar when using both the adjustment and up-and-down methods.

\section{The effect of a mnemonic device on retention of verbal material}

\author{
JAMES R. PASH and KENNETH A. \\ BLICK, University of Richmond, Va. \\ 23173
}

\begin{abstract}
After $48 h$, the retention of two lists of nine words, one list containing high meaningful words, the other low meaningful words, was compared for a group that was instructed in the use of a mnemonic device and a group that received no such instruction. The mnemonic group was instructed that the initial letters of the nine words could be arranged to spell the word "education." Overall, the high meaningful list gave superior retention, and Ss who employed the mnemonic device showed superior retention, but the general method effect was not significant.
\end{abstract}

There has been little systematic investigation concerning the alleged utility of mnemonic devices in the retention of verbal material. Senter \& Hauser (1968) evaluated a commercial technique known as the hook or peg system. The hook technique involves the overlearning of an ordered series of verbal pegs, usually common English nouns; then it becomes possible to remember a new series of words by forming images associating the new word with the previously memorized pegs. If the peg words are thoroughly overlearned, and if the associative images are sufficiently well formed, any word in the newly learned list can be recalled. Senter \& Hauser (1968) found that Ss trained with the hook system produced more correct anticipations during serial learning of $20 \mathrm{CVCs}$ than untrained Ss. Earlier, Smith \& Noble (1965) had reported similar acquisition data and noted that the peg system was more effective on retention tests with low meaningful material than with material of either high or medium meaningfulness.

The purpose of the present experiment was to examine the effectiveness of the first letter technique, a mnemonic device whereby the first letters of the words to be remembered can be arranged to spell a meaningful word. It was hypothesized that retention would be facilitated for Ss using the first letter technique, and the mnemonic device would be more effective with low meaningful material than with high meaningful material.

\section{METHOD}

The Ss were 96 students in two introductory psychology classes at the University of Richmond. One class of $56 \mathrm{Ss}$ was exposed to the mnemonic device and another class of $40 \mathrm{Ss}$ served as a control group. Within each group, approximately half of the Ss received high meaningful material and half received low meaningful material.

Two lists were prepared using 18 dissyllables selected from Noble's (1952) $m$ scale. The low meaningful list ( $m$ value $=1.89$ ) contained the following items: NEGLAN, TUMBRIL, ULNA, ICON, DAVIT, ATTAR, CAROM,
ENDIVE, and OVUM. The high meaningful list (m-value $=6.91$ ) consisted of: NAPHTHA, TYPHOON, UNCLE, INSECT, DINNER, ARMY, CAPTAIN, EFFORT, and OFFICE. The material was presented to the Ss in the form of a test booklet. The first page was a cover sheet, the second page contained the nine items in the same order as shown above, and the third page was the test sheet. At the start the Ss were told, "The purpose of this experiment is to determine whether or not you can memorize nine words in five minutes. At the end of five minutes you will be given two minutes to write as many of the words as you can remember. The words do not have to be written in the same order as they appear on the sheet." At this point, one class was told that the first letters of the nine words they were going to memorize could be arranged to spell the word "education" and that using this mnemonic device should help them remember the words. The control condition, of course, was not made aware of mnemonic technique. The Ss were not advised that they would be tested again; however, $48 \mathrm{~h}$ later they were tested for retention of the nine items.

\section{RESULTS}

Table 1 shows the nercentage correct recall for the immediate and 48-h retention tests. The columns indicate whether or not the instruction was given in the use of the mnemonic device, and the rows indicate the level of meaningfulness. The first value in the not-instructed column, 96 , means that the group correctly recalled $96 \%$ of the maximum number of correct responses for that group (162), which was determined by multiplying the number of Ss in the group $(\mathrm{N}=18)$ by the number of words to be memorized (9).

Table 2 presents the results of the statistical comparisons of the values presented in Table 1. The comparisons were made by testing for the significance of the differences between percentages, with the first eight comparisons for uncorrelated data and the last four for correlated data. All tests were of the one-tail variety at the $1 \%$ level of significance. Note that the third and fourth comparisons indicate that, even after $48 \mathrm{~h}$, instruction in the use of the mnemonic device did not significantly improve retention of either high or low meaningful lists. As was expected, there was a significant retention loss over $48 \mathrm{~h}$, as shown by the last four comparisons, and high meaningful material was retained at a significantly higher level than low meaningful material at $48 \mathrm{~h}$, as shown by Comparisons 7 and 8.

\section{DISCUSSION}

The original hypothesis concerning the 